\title{
Range of Motion Exercise Suppresses Myofibroblast Proliferation in the Joint Capsule in a Rat Joint Contracture Model
}

Taro Matsuzaki ( $\nabla$ tarosan@mhs.mp.kanazawa-u.ac.jp )

Kanazawa University

Shinya Yoshida

Kanazawa University

Masahiro Hoso

Kanazawa University

\section{Research Article}

Keywords: motion exercises, Eighteen male Wistar rats, inhibit myofibroblast proliferation

Posted Date: March 1st, 2021

DOl: https://doi.org/10.21203/rs.3.rs-244641/v1

License: (c) (i) This work is licensed under a Creative Commons Attribution 4.0 International License.

Read Full License 


\section{Abstract}

This study aimed to perform range of motion exercises on a rat knee joint contracture model and clarify changes in the joint range of motion and number of myofibroblasts in the joint capsule. Eighteen male Wistar rats were used and randomly divided into the following three groups: control, immobilized, and exercise. The right hindlimb knee joints of rats in the immobilized and exercise groups were immobilized, and the animals in the exercise group started the range of motion exercises the day after the joint immobilization. After the two weeks experimental period, the range of knee joint extension angle was measured, and the knee joint was collected. To observe the posterior joint capsule of the rat knee joint, hematoxylin and eosin staining and immunostaining were performed. Differences in knee extension restriction angles were significant between all groups, and differences in the number of alpha smooth muscle actin-positive cells were significant between the control and immobilization groups. These results indicate that joint immobilization leads to myofibroblast proliferation, whereas gentle exercise that does not maintain sufficient range of motion in the joint may inhibit myofibroblast proliferation.

\section{Introduction}

The joint capsule is a joint component that can be cited as a cause of limited joint range of motion (ROM). Several reports on experimentally creating joint contracture models using animals and investigating changes in joint components have been reported since the 1960s [1-3]. We have immobilized rat hind limbs in various ways and observed joint structure changes [4-7], and reported that collagen fibers become dense in the posterior joint capsule. In recent years, reports have focused on the myofibroblasts in joint capsule inducing the joint capsule fibrosis $[8,9]$. These experiments indicate that joint immobilization leads to increased number of myofibroblasts in the joint capsule, suggesting this as the cause of joint capsule fibrosis. An important purpose of physical therapy is the prevention and treatment of joint ROM limitations. In the clinical environment, joint contracture treatment includes hyperthermia and therapeutic gymnastics in addition to ROM exercises (ROM Ex); however, the treatment effectiveness can only be determined by measuring the joint ROM. ROM Ex is a physical therapy treatment technique evaluated only by the joint ROM, and there are no reports on how the tissue changes. Joint immobilization causes decreased joint ROM, which is largely due to muscle shortening and rigidity up to 2 weeks, and, thereafter, due to many joint structures (cartilage, joint capsule, ligaments, etc.). Previous reports showed that myofibroblasts are found in the joint capsule due to joint immobilization or joint capsule trauma $[8,10-12]$. Since myofibroblasts are involved in tissue fibrosis, they are thought to cause physical hardening of the joint capsule. Therefore, this study aimed to perform ROM Ex on a rat knee joint contracture model and clarify joint ROM and myofibroblast changes in the joint capsule.

\section{Results}

All rats survived the experimental period without symptoms of infection. Table 1 shows the knee joint limitation and number of a-SMA-positive cells. The knee joint extension limit angles were $30.3 \pm 2.9$ degrees, $69.0 \pm 3.7$ degrees, and $41.7 \pm 7.8$ degrees in the control, immobilized, and exercise groups, 
respectively. The differences among all groups, described as mean difference ( $95 \%$ confidence interval [CI]), were significant as follows: between the control and immobilized groups, 38.7 [28.8-48.6], $p=1.6$ 12e; between the control and exercise groups, 17.0 [8.2-25.8], $p=1.8-8 \mathrm{e}$; and between the exercise and immobilized groups, 21.7 [11.0-32.4], $p=6.9-9 e$ ). Conversely, the number of a-SMA-positive cells was $3.7 \pm 1.4,12.7 \pm 6.1$, and $4.8 \pm 1.7$ in the control, immobilized, and exercise groups, respectively. Differences (shown as mean difference [95\% Cl]) were significant between the control and immobilized groups, 9.0 [2.8-15.2], $p=0.002$ ); and between the exercise and immobilized groups, 7.9 [1.6-14.2], $p=$ 0.006). However, no significant difference was observed between the control and exercise groups (7.9 [$1-3.4], p=0.851)$.

\section{Histopathological changes in the posterior joint capsule}

Each joint capsule is shown in Fig. 2. In the posterior capsule, a collagen fiber bundles were arranged in the same direction in the control group, and a relatively loose image was observed with a gap between the fiber bundles. On the other hand, in the posterior capsule of the immobile group, the number of fibroblasts tends to increase, and infiltration of activated fibroblasts (indicating cell plumping) were observed. And collagen fiber bundles were somewhat rigid, interfiber bundles were narrow and dense. In the exercise group, collagen fiber bundles were kept loose, but the fiber orientation was not uniform and the cell density tended to be high. An increase in fibroblasts was observed compared to the control group, but not as much as in the immobile group. Infiltration of inflammatory cells (neutrophils) was also observed in the tissue.

\section{Discussion}

Joint contracture is one of the common disorders associated with joint Immobility, which often interferes with daily activities. And physiologists often perform ROM Ex. as a treatment for joint contractures. To clarify its pathology, several studies have been conducted by fixating the joints of animals and examine the joint histology. Various methods such as cast fixation [13-18], surgery with internal fixation [1, 3, 19, $20]$, and external fixation $[2,20,21]$ have been used to perform joint immobilization; however, in this study we used external fixation. External fixation facilitates easy joint immobilization or fixation removal to move the joint; however, a risk of infection is present. Therefore, sterilization should be carefully performed during the intervention, and the experiments should be performed while preventing infection. In our experiments, infection appeared to be well controlled. The myofibroblast was initially identified by means of electron microscopy in granulation tissue of healing wounds as a modulated fibroblast, exhibiting features of smooth muscle cells $[22,23]$. Moreover, they are observed in normal and pathological tissues. They are considered to be present in fibrous lesions such as wound healing and cirrhosis in pathological tissues [24]. In these experiments, the a-SMA antibody that stains smooth muscle actin was used. Among the tissues of the joint capsule, vascular pericytes are known as tissues having smooth muscle actin in addition to myofibroblasts. To rule out vascular pericytes, vascular endothelial cells adjacent to vascular pericytes were stained with CD34 antibody. We excluded cells adjacent to CD34 antibody-positive cells among a-SMA antibody-positive cells. The results distinguished 
myofibroblasts, which have been shown to play an important role in post-injury tissue reconstruction and pathological changes characterized by fibrosis [25]. Hildebrand et al. reported that immobilization of the hind limb of rabbit increases myofibroblast production in the joint capsule [8], and also reported that myofibroblasts increased in the joint capsule of the human elbow joint with a limited ROM due to trauma [26]. This result indicates that joint immobility leads to myofibroblast hyperplasia and generic healing mechanism. With regard to ROM Ex in these experiments, the ROM limitation was significantly smaller in the exercise group than in the immobilized group, suggesting that ROM Ex was effective. Trudel et al. reported that within 2 weeks after joint immobilization, the ROM is limited by muscles due to joint components [27], suggesting that the ROM Ex protocol conducted may not have been sufficient as a treatment for muscle shortening. Conversely, the number of a-SMA-positive cells in the joint capsule was not different between the exercise and control groups, and a significant increase was observed only in the immobilized group. These results suggest that joint capsule immobilization leads to myofibroblasts proliferation; however, mild exercise to maintain the ROM of the joint may inhibit myofibroblasts proliferation.

The limitations of this experiments were the short experiment period (only 2 weeks), and other periods were not considered, and the fact that only one ROM Ex protocol was performed; therefore, factors that induce changes in strength or frequency were not investigated. Darby et al. reported that microfilaments increased from day 6 to day 15 after injury in a rat wound healing model, but progressively regressed thereafter, and disappeared by day 30[28]. For this reason, the duration of this experiment seems appropriate; however, changes at weeks 1 or 4 should still be determined. In addition, the mechanism of myofibroblasts proliferation when joints are immobilized remains to be elucidated; however, why exercise can suppress their proliferation is still unclear. In conclusion, this experiment suggests that joint movement may inhibit joint capsule fibrosis, and that not only muscle stretching but also joint movement is necessary to maintain the ROM.

\section{Materials And Methods}

\section{Experimental animals and animal care}

The protocol for these studies was approved by the Animal Care Committee and Institutional Ethics Committee of Kanazawa University (approval no.

153504), and conducted in accordance with the ARRVE guidelines [29] and all procedures for animal care and treatment were performed in accordance with the guidelines for the care and use of laboratory animals at Kanazawa University. In total, eighteen 9-week-old Wistar male rats (body weight 243-275 g) were used in this study. The rats were purchased (Sankyo Labo Service Corporation, INC.; Toyama, Japan) at 8 weeks of age and placed in individual cages, and the experiment was started after acclimatization to the environment over a week. The breeding room temperature was kept constant, and the lighting was turned on and off every $12 \mathrm{~h}$. Food and water were given ad libitum.

\section{Joint immobilization and range of motion exercise}


Rats were randomly divided into the following three groups $(n=6)$ : control, immobilized, and exercise. The right hindlimb knee joints of rats in the immobilized and exercise groups were immobilized in a $120^{\circ}$ flexion position using external fixation under general anesthesia with an intraperitoneal injection of sodium pentobarbital according to a previous study [30]. Joint immobilization was performed under clean condition with the sterilized instruments. After the intervention, the immobilized and exercise groups were able to move freely within the cage with their hindlimbs immobilized, and no drinking and eating problems were observed. The exercise group started the ROM Ex under general anesthesia from the day after that of the intervention. Prior to the ROM Ex, the rat trunk was fixed under general anesthesia as in a previous study [30], and the external fixation was removed. Thereafter, a force gauge was attached to the foot of the animals, and the animal's foot was pulled through the force gauge at $1 \mathrm{~N}$ to the caudal direction for the first 5 seconds to extend the knee joint, and the next $5 \mathrm{~s}$ were held manually at $120^{\circ}$ flexion. This exercise was performed once a day for $6 \mathrm{~min}$ for 7 days a week. The joints of the rats in the immobilized group were immobilized, and the animals were housed without any other intervention, whereas the control group was housed without any intervention. The experimental period lasted 2 weeks as in previous studies of our group [30-32]. At the end of the experimental period, all rats were euthanized by overdose following intraperitoneal injection of pentobarbital sodium. The knee joint ROM was immediately measured according to a previous study [30], and then the hip joint was cut off and the right hind limb was collected as a specimen. The hind limb was fixed with a neutral buffered formalin solution and then decalcified with a Plank Rychlo solution. After decalcification, the knee joint was sagittal in the middle and neutralized with $5 \%$ sodium sulfate solution. Tissue fixation, decalcification, and neutralization were all carried out for $72 \mathrm{~h}$ at $4^{\circ} \mathrm{C}$. After neutralization, the sample was washed with water and degreased/dehydrated with ethanol, and embedded in paraffin wax. Paraffin-embedded sections were sliced at $3 \mu \mathrm{m}$ at a site where the posterior joint capsule could be observed and tissue slides were prepared. To histopathological observation for the posterior joint capsule of the rat knee joint, specimens were stained hematoxylin and eosin. Tissues were analyzed using a light microscope (BX-51; Olympus Corporation, Tokyo, Japan) and a digital camera (DP-74 and cellSens (ver.2.3); Olympus Corporation).

\section{Immunostaining}

To detect myofibroblasts at joint capsule, immunostaining was performed using mouse anti-alpha smooth muscle actin (a-SMA) antibody (ab119952; Abcam plc, Cambridge, UK). However, within the joint capsule, cells that reaction to a-SMA antibody include not only myofibroblasts but also pericytes of blood vessels. To distinguish between myofibroblasts and vascular pericytes, a rabbit anti-CD34 antibody (ab81289; Abcam plc) was used to stain vascular endothelial cells adjacent to vascular pericytes. Tissue slides were deparaffınized, hydrated with graded alcohol, and washed with phosphate-buffered saline (PBS) for $5 \mathrm{~min}$. Next, the tissue slides were placed in sodium citrate buffer (10 mM, pH 6.0) and heated for $60 \mathrm{~min}$ at $75^{\circ} \mathrm{C}$ for antigen retrieval. After slow cooling to room temperature, endogenous peroxidase was inactivated with a $1 \%$ solution of hydrogen peroxide in PBS for 20 min at room temperature. In order to block nonspecific antibody binding, the sections were incubated with Protein Block Serum-Free (X0909; Dako Japan, Tokyo, Japan) for $15 \mathrm{~min}$ at room temperature. Then, the tissue slides were incubated 
overnight at room temperature with a mixture of mouse anti-a-SMA antibody (1:400 dilution) and rabbit anti-CD34 antibody (1:400 dilution). On the next day, after rinse the tissue slides with PBS, slides were incubated with the appropriate secondary antibodies. First, Histofine Simple Stain AP (M) (for mouse primary antibodies, labeled with alkaline phosphatase, 414241; Nichirei Biosciences Inc., Tokyo, Japan) was dropped on the slides and incubated for 60 minute. Subsequently, a-SMA antibody positive cells were stained blue using the VECTOR® Blue alkaline phosphatase substrate kit III (Cat. No, SK-5300; Vector Laboratories). The Vector Blue staining solution was prepared by adding the reagent to $5 \mathrm{ml}$ of $100 \mathrm{mM}$ Tris-HCl buffer ( $\mathrm{pH}$ 8.5) according to the instructions and stirring well. Then, the staining solution were dropped on the slide and incubated for about 10 minutes while confirming with an optical microscope. After rinsing tissue slides with PBS, Histofine Simple Stain Rat MAX PO (R) (for rabbit primary antibodies, labeled with peroxidase, 414181; Nichirei Biosciences Inc.) were used as secondary antibodies and dropped on the slides and incubated for 60 minutes. After incubation, rinse tissue slides in PBS, subsequently CD34 antibody-positive tissues were stained using the VECTOR® NovaRED ${ }^{\mathrm{TM}}$ peroxidase substrate kit (Cat. No. SK-4800; Vector Laboratories, California, USA). The NovaRED staining solution was prepared as instructed, and stirring them well. The Working solution was dropped on the tissue slide, incubated for 10 minutes while observing with a light microscope, and then washed with PBS. The tissue was counterstained with Nuclear-fast-red, washed with water, dehydrated with ethyl alcohol, clarified with NEO-CLEARß(Merck KGaA, Darmstadt, Germany), and then mounted with NeoMount $^{\text {TM }}$ (Merck KGaA, Darmstadt, Germany).

\section{Cell number counting}

After staining, the slides were observed with an optical microscope, and images were taken with a digital camera. The number of stained cells in $2 \mathrm{~mm} 2$ of the joint capsule was counted. While counting the number of cells, in order to exclude vascular pericytes from the cells stained blue with a-SMA antibody, cells suspected to be blood vessels and cells adjacent to CD34-positive cells (stained red) were excluded (Fig. 1). Cell counting was performed blindly.

\section{Statistical analysis}

Knee joint limitation data and a-SMA-positive cell counts were each analyzed with one-way analysis of variance using IBM SPSS Statistics for Windows (version 25; IBM Corp., Armonk, NY, USA) and Bonferroni post hoc multiple comparisons. Significant differences were considered at $p<0.05$, and all results were reported as mean \pm SD values.

\section{Declarations}

\section{Acknowledgment}

The authors thank the members of Human Pathology at the Kanazawa University Graduate School of Medicine for their advice on specimen preparation and staining techniques. 
We gratefully thank Ms. Ami lkeda for helping with the experiment.

\section{Funding}

This experiment was supported by JSPS KAKENHI Grant-in-Aid for Scientific Research(C) Number JP16K01450.

\section{Author Contributions}

Conceptualization: TM, SY

Data curation: $\mathrm{TM}, \mathrm{MH}$

Formal analysis: $\mathrm{TM}, \mathrm{MH}$

Funding acquisition: $\mathrm{TM}, \mathrm{MH}$

Investigation: TM, SY, MH

Methodology: TM, SY, MH

Project administration: $\mathrm{TM}, \mathrm{MH}$

Resources: TM, MH

Software: TM

Supervision: $\mathrm{MH}$

Validation: TM, SY, MH

Visualization: TM, SY, MH

Writing- Original draft: TM

Writing- review\& editing: TM, SY, MH

\section{Competing interest statement}

The authors have no conflicts of interest to report.

Data availability. All relevant data are within the paper.

\section{References}

1 Evans, EB., Eggers, GWN., Butler, JK.\& Blumel, J. Experimental Immobilization and Remobilization of Rat Knee Joints. J Bone Joint Surg Am.42, 737-758 (1960). 
2 Trias, A. Effect Of Persistent Pressure on the Articular Cartilage. J Bone Joint Surg Br.43, 376-386 (1961).

3 Peacock EE Jr. Some Biochemical and Biophysical Aspects of Joint Stiffness: Role of Collagen Synthesis as Opposed to Altered Molecular Bonding. Ann Surg.164, 1-12 (1966).

4 Matsuzaki,T. et al. Histopathological Changes of the Intermuscular Adipose Tissue of the Rat Thigh during Experimental Knee Joint Contracture. Rigakuryoho Kagaku.24, 901-905 (2009) (in Japanese)

5 Watanabe, M., Hoso, M., Yoshikubo, H., Matsuzaki, T. \& Kojima, S. Histopathological Effects of the Stretching on Joint Capsule after Contracture; A study of Knee Joint Immobility Model in the Rat. Rigakuryoho Kagaku.24, 403-409 (2009) (in Japanese)

6 Watanabe, M., Hoso, M., Hibino, I., Matsuzaki, T. \& Kojima, S. Histopathological Changes of Joint Capsule after Joint Immobility Compared with Aging in Rats. J. Phys. Ther. Sci.22, 369-374 (2010).

7 Matsuzaki, T., Kojima, S., Watanabe, M., Yoshida, S. \& Hoso, M. Histopathological Changes in the Infrapatellar Fat-Pad of Rats with Knee Contracture. Ishikawa Journal of Physical Therapy. 12, 11-14 (2012) (in Japanese)

8 Hildebrand, KA., Sutherland, C. \& Zhang, M. Rabbit knee model of post-traumatic joint contractures: the long-term natural history of motion loss and myofibroblasts. J Orthop Res. 22, 313-320 (2004).

9 Sato, Y. et al. Resolution of liver cirrhosis using vitamin A-coupled liposomes to deliver siRNA against a collagen-specific chaperone. Nat. Biotechnol. 26, 431 (2008).

10 Abdel MP. et al. Myofibroblast cells are preferentially expressed early in a rabbit model of joint contracture. J Orthop Res.30, 713-719 (2012).

11 Doornberg, JN. et al. Temporary presence of myofibroblasts in human elbow capsule after trauma. J Bone Joint Surg Am. 96, 10.2106/JBJS.M.00388 (2014).

12 Sasabe, R. et al. Effects of joint immobilization on changes in myofibroblasts and collagen in the rat knee contracture model. J Orthop Res.35, 1998-2006 (2017).

13 Videman, T. Experimental Osteoarthritis in the Rabbit Comparison of Different Periods of Repeated Immobilization. Acta Orthop Scand.53, 339-347 (1982).

14 Behrens, F., Kraft, EL. \& Oegema, TR. Jr. Biochemical Changes in Articular Cartilage after Joint Immobilization by Casting or External Fixation. J Orthop Res. 7, 335-343 (1989). 
15 Kiviranta, I. et al. Articular cartilage thickness and glycosaminoglycan distribution in the young canine knee joint after remobilization of the immobilized limb. J Orthop Res.12, 161-167 (1994).

16 Haapala, J. et al. Remobilization does not fully restore immobilization induced articular cartilage atrophy. Clin Orthop Relat Res. 362, 218-229 (1999).

17 Leroux, MA. et al. Altered mechanics and histomorphometry of canine tibial cartilage following joint immobilization. Osteoarthr Cartilage. 9, 633-640 (2001).

18 Booth, FW. \& Kelso, JR. Production of rat muscle atrophy by cast fixation. J. Appl. Physiol.34, 404406 (1973).

19 Thaxter, TH., Mann, RA. \& Anderson, CE. Degeneration of Immobilized Knee Joints in Rats Histlogical and Autoradiographic Study. J Bone Joint Surg Am 47, 567-585 (1965).

20 Tart, RP. \& Dahners, LE. Effects of electrical stimulation on joint contracture in a rat model. $J$ Orthop Res. 7, 538-542 (1989).

21 Pitsillides, AA., Skerry, TM. \& Edwards, JC. Joint immobilization reduces synovial fluid hyaluronan concentration and is accompanied by changes in the synovial intimal cell populations. Rheumatology (Oxford).38, 1108-1112 (1999).

22 Gabbiani, G., Ryan, GB. \& Majne, G. Presence of modified fibroblasts in granulation tissue and their possible role in wound contraction. Experientia. 27, 549-550 (1971).

23 Desmoulière, A., Chaponnier, C. \& Gabbiani, G. Tissue repair, contraction, and the myofibroblast. Wound Repair Regen. 13, 7-12 (2005).

24 Ina, K. et al. Transformation of interstitial fibroblasts and tubulointerstitial fibrosis in diabetic nephropathy. Med Electron Microsc.35, 87-95 (2002).

25 Hinz, B. \& Gabbiani, G. Mechanisms of force generation and transmission by myofibroblasts. Curr Opin Biotechnol. 14, 538-546 (2003).

26 Hildebrand, KA., Zhang, M., van Snellenberg, W., King, GJ. \& Hart, DA. Myofibroblast numbers are elevated in human elbow capsules after trauma. Clin Orthop Relat Res.419, 189-197 (2004).

27 Trudel, G. \& Uhthoff, HK. Contractures Secondary to Immobility: Is the Restriction Articular or Muscular? An Experimental Longitudinal Study in the Rat Knee. Arch Phys Med Rehabil.81, 6-13 (2000).

28 Darby, I., Skalli, O. \& Gabbiani, G. Alpha-smooth muscle actin is transiently expressed by myofibroblasts during experimental wound healing. Lab Invest. 63, 21-29 (1990).

29 Percie du Sert N. et al. Reporting animal research: Explanation and elaboration for the ARRIVE guidelines 2.0. PLoS Biol.18,e300041: 10.1371/journal.pbio.3000411(2020). 
30 Matsuzaki, T., Yoshida, S., Kojima, S., Watanabe, M. \& Hoso, M. Influence of ROM Exercise on the Joint Components during Immobilization. J Phys Ther Sci.25, 1547-1551 (2013).

31 Yoshida, S. et al. Histopathological changes in the periphery of the sciatic nerve of rats after knee joint immobilization. J Phys Ther Sci.25, 623-626 (2013).

32 Matsuzaki, T., Yoshida, S., Ikeda, A. \& Hoso, M. Changes in joint components after knee immobilization associated with hindlimb unweighting in rats. J Wellness Health Care. 42, 33-40 (2019).

\section{Tables}

Table 1. Range of motion of the knee joints (A) and number of $\alpha$-SMA antibody positive cells in each groups (B).
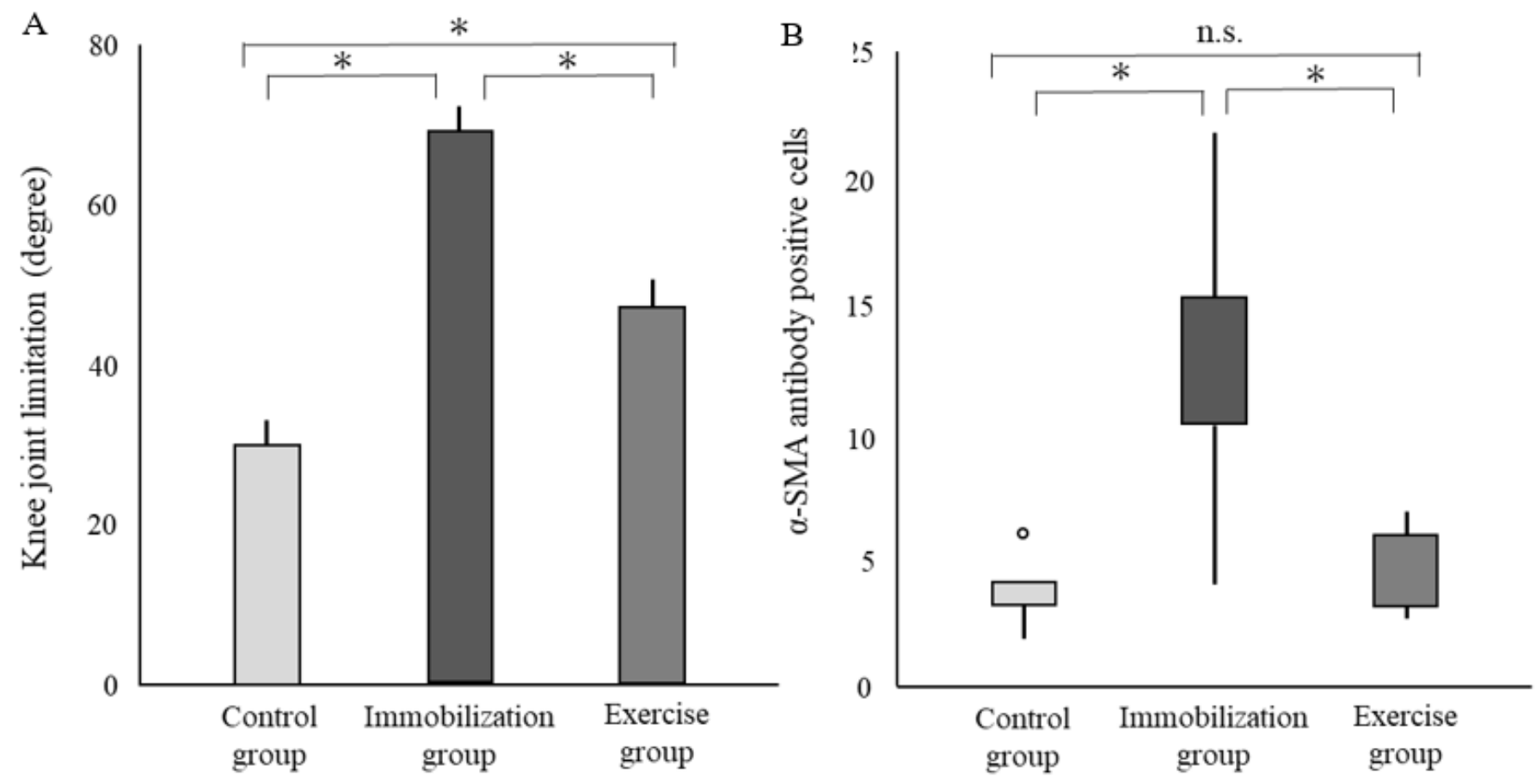

$\left({ }^{*}\right.$ means $\left.\mathrm{p}<0.05\right)$

\section{Figures}


A

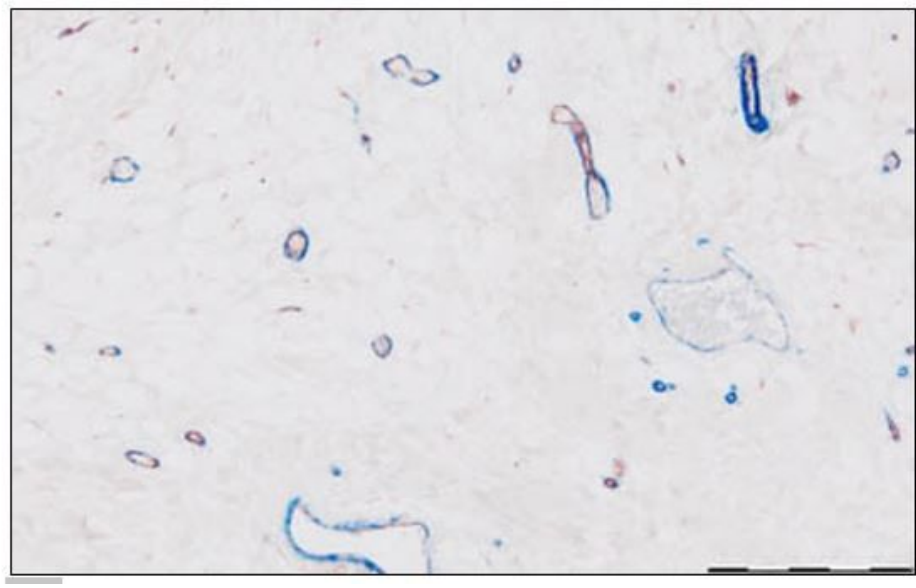

B

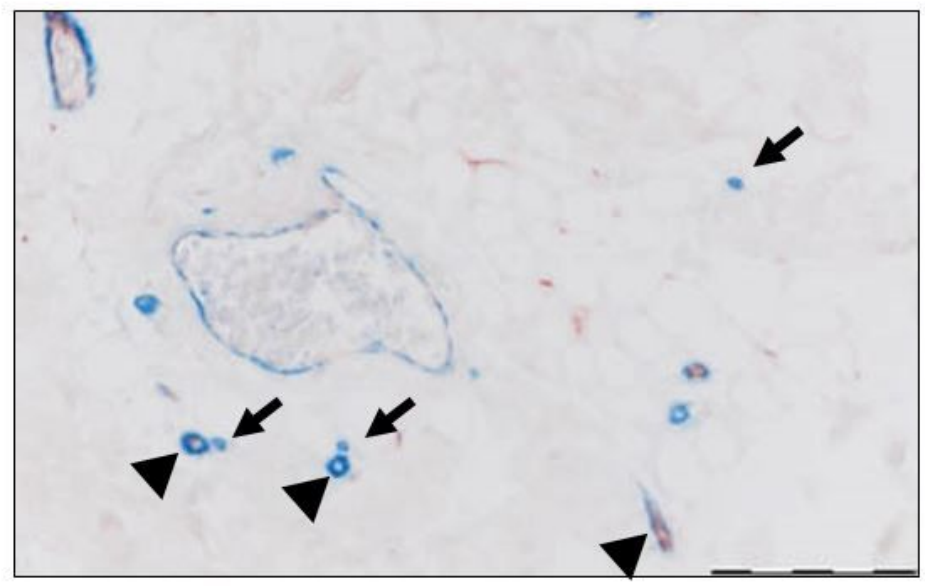

\section{Figure 1}

Posterior joint capsule used for cell count measurement. A: Double immunostaining was performed to differentiate between vascular pericytes and myofibroblasts. Anti-CD34 antibody positive cells (vascular endothelial cells) are stained red, and anti-a-SMA antibody positive cells (smooth muscle cells) are stained blue. Scale bar $=200 \mu \mathrm{m}$ B: High-power view of A. Among the blue-stained anti-a-SMA antibodypositive cells, cells thought to be pericytes $(\boldsymbol{\Lambda})$ were excluded, and cells not adjacent to vascular endothelial cells $(\rightarrow)$ were counted. Scale bar $=100 \mu \mathrm{m}$ 

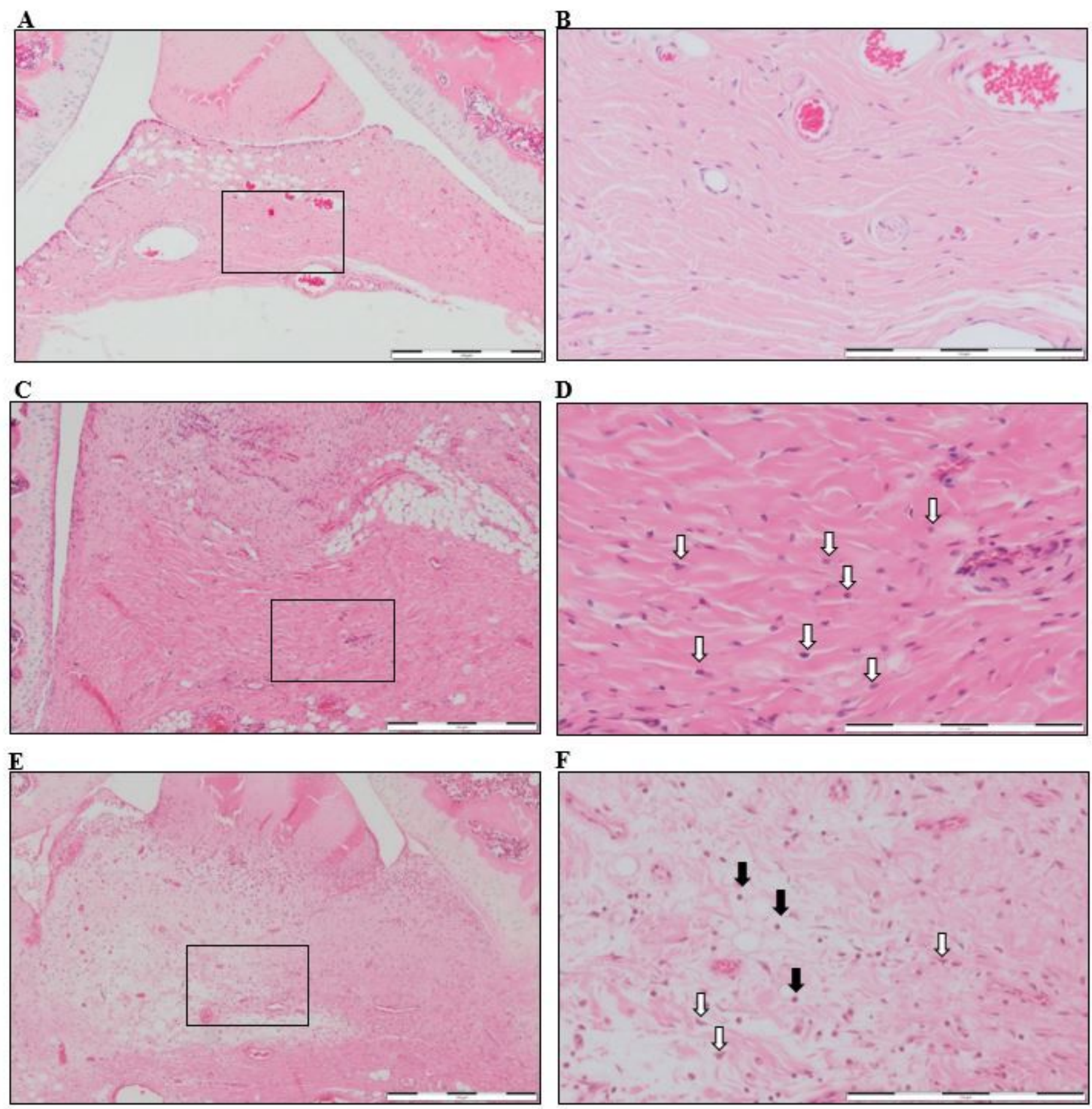

\section{Figure 2}

Histopathological assessment of regions in the posterior joint capsule. In the control group (Fig.2A and 2B), the collagen fiber bundles overlap in the same direction. There are gaps between the fiber bundles and no densification is observed. In the immobilized group (Fig.2C and 2D), coarse fibrous tissue, loss of interfiber spaces, and activated fibroblasts (showing cell plumping) were observed in the joint capsule (white arrows). Also, inflammatory cells (neutrophils, black arrows) infiltration was observed in the tissue. In the exercise group (Fig.2E and 2F), collagen fiber densification were also observed, but it was milder than that of the immobile group. Activated fibroblasts were observed and also inflammatory cells 


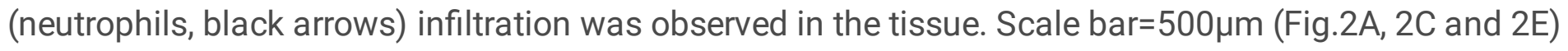
and $200 \mu \mathrm{m}$ (Fig.2B, 2D and 2F). 